\title{
Pressure dependence of the dielectric loss minimum slope for ten molecular liquids
}

\author{
A.I. Nielsen ${ }^{\mathrm{a} *}$, S. Pawlus ${ }^{\mathrm{b}}$, M. Paluch ${ }^{\mathrm{b}}$ and J.C. Dyre ${ }^{\mathrm{a}}$ \\ ${ }^{a}$ DNRF Centre 'Glass and Time', IMFUFA, Department of Sciences, Roskilde, Denmark; \\ ${ }^{b}$ Institute of Physics, Silesian University, Katowice, Poland
}

(Received 23 May 2008; final version received 5 November 2008)

\begin{abstract}
We present a comprehensive study of data for the dielectric relaxation of ten glass-forming organic liquids at high-pressure along isotherms, showing that the primary $(\alpha)$ high-frequency relaxation is well-characterized by the minimum slope and the width of the loss peak. The advantage of these two parameters is that they are model independent. For some materials with $\beta$ processes in the $\mathrm{mHz}$ and $\mathrm{kHz}$ range, the high-frequency slope tends to be $-\frac{1}{2}$ with pressure increase. In addition, the two parameters capture the relaxation shape invariance at a given relaxation time but different combinations of pressure and time.
\end{abstract}

Keywords: compression; time pressure temperature super position; minimum slope; width; dielectric

Glass may be regarded as the fourth state of conventional matter: isotropic as the liquid state, but solid as the crystalline state. With the notable exception of helium, any liquid may be turned into glass by cooling it fast enough to avoid crystallization [1-5].

Physical systems usually relax following perturbations forced upon them. The relaxation of the systems consists of processes going on at different time-scales. The dominant and slowest relaxation process of a glass-forming liquid is the so-called $\alpha$ process. The $\alpha$ process defines the liquid relaxation time, an important quantity because the glass transition takes place when the relaxation time significantly exceeds the inverse relative cooling rate. Compression of supercooled liquid slows down the $\alpha$ relaxation (increases the characteristic relaxation time, $\tau$ ). On the other hand, this effect can be compensated by heating up the liquid. Different combinations of $p$ and $T$ can result in the same relaxation dynamics at the same $\tau$, or materials obey the temperature-pressure superposition at the same relaxation times (TTPS) [6-11]. There are different kinds of secondary $(\beta)$ relaxation, including Johari-Goldstein $(\mathrm{JG})$ and those of intramolecular motions [11-16] or any excess wings.

In a paper from 2001, it was shown that the high-frequency slope of the dielectric loss for a group of materials tends to be $-\frac{1}{2}$ as the temperature approaches the glass transition temperature, $T \rightarrow T_{\mathrm{g}}$, and this was linked to time-temperature superposition (TTS) [17].

*Corresponding author. Email: albenan@ruc.dk 
But a general prevalence of -0.5 for the slope has been found in highly viscous liquids, no matter whether TTS is obeyed [18]. In those papers, the dielectric scans were taken at ambient pressure. In the following, we investigate whether this result holds for dielectric frequency scans for ten liquids along isotherms with increasing pressure $\left(p \rightarrow p_{\mathrm{g}}\right)$, and if the model-independent shape quantities, minimum slope and half loss peak width, capture TTPS. The minimum slope, of course, may well be affected by secondary processes, well expressed in the experimental frequency window as well as the underlying low-frequency $\beta$ relaxation (or an excess wing), but no attempt is made to compensate for these effects. The point is that the minimum slope is an objective shape parameter.

We have measured the dielectric loss, $\varepsilon^{\prime \prime}$, as a function of frequency for a number of organic glass formers slightly below the glass transition pressure. In order to avoid bias, data were selected prior to their analysis. A model-independent data analysis was performed; i.e. without fitting data to any of the standard functions (stretched exponential, Havriliak-Negami, Cole-Cole, Cole-Davidson, etc.). Thus, there is no need to distinguish between liquids with and without clearly resolved secondary $(\beta)$ processes. Very accurate data are required in order to obtain reliable slopes by numerical differentiation. The selection criteria were low noise, well-defined loss peak, and sufficient length of the high-frequency part of the dissipation in order to find the minimum slope. In order to avoid bias, data were selected prior to analysis. The data analysis was automated as far as possible via Matlab programs [18].

Moreover, following the philosophy of making as direct and unbiased data analysis as possible, no attempt was made to subtract possible contributions from DC conductivity. Of course, if the conductivity overruled the primary relaxation, then the particular scan was excluded from the analysis. In order to avoid the influence of the conductivity, a frequency scan was discarded if one of the following points was not fulfilled: the lowfrequency slope is 1 (within the measure noise); or TTS around the loss peak is obeyed (of course, in a range where $\beta$ relaxation does not interfere). ${ }^{1}$

The chemicals are chlorinated biphenyl (CBP, Aroclor [16]), 1,1'-bis(p-methoxyphenyl) cyclohexane (BMMPC, [19]), Di-iso-butyl phthalate (DisoBP, [20]), di-propylene glycol (DPG, [21]), tri-propylene glycol (TPG, [10]), diglycidyl ether of bisphenol-A (EPON828, [21]), propylene carbonate (PC, [8]), phenolphthalein-dimethylether (PDE [23]), tetramethyltetra-phenyltrisiloxane (DC704, this work, Dow Corning $704^{\circledR}$ diffusion pump fluid), perhydroisoquinoline (decahydroisoquinoline, PHIQ, this work, 99\%, Aldrich, Figure 1b). DC704 is represented in Figure 1a with three isotherms $T=253 \mathrm{~K}$, $p \in[6$; 2460] bar, $T=263 \mathrm{~K}, p \in[1333$; 2404] bar and $T=283 \mathrm{~K}, p \in[2559$; 2404] bar, and PHIQ with two isotherms in Figure $1 b, T=232 \mathrm{~K}, p \in[0 ; 1000]$ bar and $T=293 \mathrm{~K}$, $p \in[3375 ; 17500]$ bar. The measurements were carried out on the set-up described in $[24,25]$. The pressure-transmitting liquid was a silicon oil. The measure cell consisted of a capacitor - two parallel parts of the steel cylinder, separated by Teflon stripes and kept together by two Teflon rings - placed into a tightly closed Teflon container filled up with the sample liquid. ${ }^{2}$

The low-frequency (long-time) properties of the $\alpha$ process are fairly trivial; the vast majority of glass-forming liquids here exhibit what corresponds to a cut-off in the relaxation time distribution function at long-times [25-27]. Focusing on the short-time (high-frequency) relaxation properties, at each temperature and pressure we identified the minimum slope in the standard $\log -\log$ plot, $\alpha_{\min } \equiv \min \left(d \log \varepsilon^{\prime \prime} / d \log f\right)<0$, where $f$ is the frequency and log is the base-10 logarithm (see Figure 2a). This identifies the inflection 

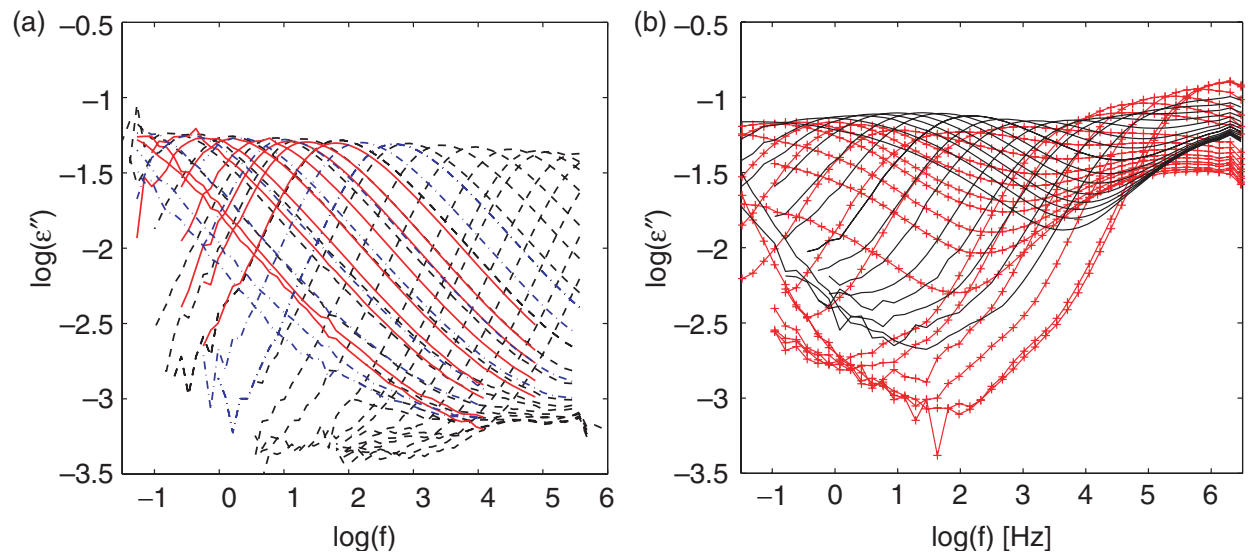

Figure 1. (Color online). Full dataset $\log -\log$ plot of the dielectric loss $\varepsilon^{\prime \prime}$ as a function of frequency for (a): DC 704 at $T=253 \mathrm{~K}, p \in[6 ; 2460]$ bar (black --), $T=263 \mathrm{~K}, p \in[1333 ; 2404]$ bar (red -), and $T=283 \mathrm{~K}, p \in[2559 ; 2404]$ bar (blue - . - ). There is a secondary process at $0.1-1 \mathrm{MHz}$ that is relatively pressure insensitive. (b): PHIQ at $T=232 \mathrm{~K}, p \in[0 ; 1000]$ bar (black -. - ) and $T=293 \mathrm{~K}$, $p \in[3375 ; 17500]$ bar $($ red + ). The scan is characterized by a well-resolved pressure-independent $\beta$ process with relatively high amplitude at $1 \mathrm{MHz}$. Only a few of the presented are used in the analysis.

(a)

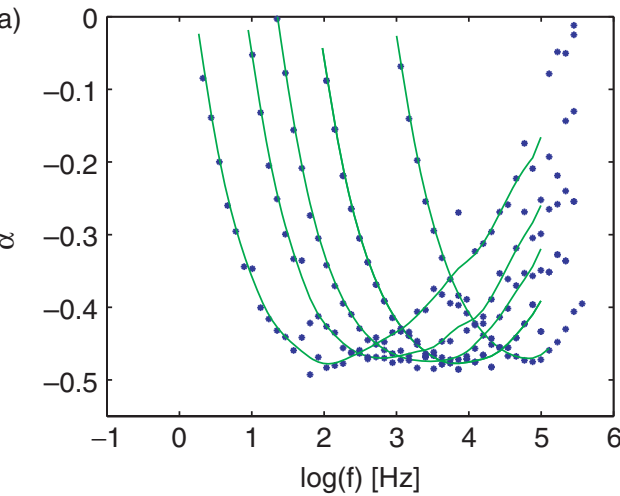

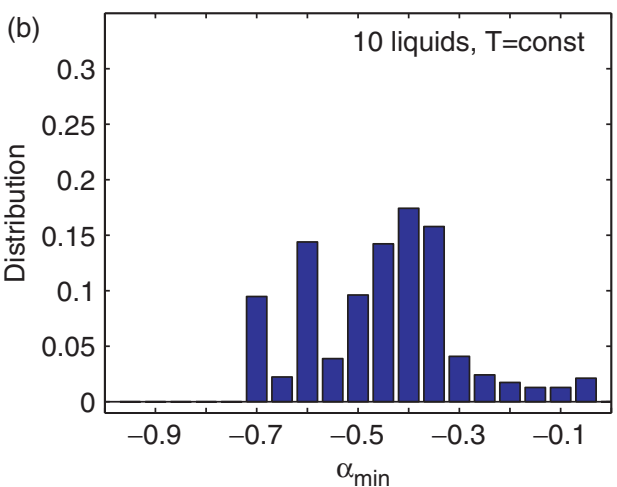

Figure 2. (Color online). (a): The slope of the double logarithmic dielectric dissipation (with stars) at some chosen pressures from the data in Figure 1a: DC704 at 253K. The green line indicates the averaged values that are used to find the minimum slope, $\alpha_{\min }$. (b): Histogram of the minimum-slope distribution for the ten organic glass-forming liquids (one column for $-0.85<\alpha_{\min }<-0.75$, one for $-0.75<\alpha_{\min }<-0.65$, etc.). Since the number of pressures investigated varies from isotherm to isotherm and the number of isotherms for each liquid, each minimum slope observation is weighted by a factor $1 /(N n) . N$ is the number of data points (pressures) in a dataset for the given liquid at the given temperature, and $n$ is the number of isotherm datasets for the given liquid. In this way, all liquids contribute equally to the histogram. Note that an isotherm may contribute to more than one column in this figure, since $\alpha_{\min }$ vary with pressure.

point above the loss-peak frequency. The number $\alpha_{\min }$ gives the best approximate inverse power-law description of the loss decay above the peak: $\varepsilon^{\prime \prime}(f) \propto f^{-\left|\alpha_{\min }\right|}$ applies to a good approximation over a significant frequency range. Only data with a well-defined minimum slope or a clear slope plateau in the point-by-point numerical differentiation were used. 
If relatively low noise appears in the derivative, a smoothing is obtained by averaging the values (Figure 2a) and the minimum value found. The averaging was done to maximize the number of datasets. The distribution of the minimum slopes for the ten different liquids for all temperatures and frequency-pressure scans is presented in Figure $2 \mathrm{~b}$. The above-mentioned limitations, as well as the different pressure and frequency ranges and intervals, imply that the number of datasets (isotherms) per liquid varies from one to three, and the number of data points (pressures) for each dataset at constant temperature for a liquid varies from four up to 26 . To compensate for this, we give equal weight to each liquid in Figure 1b. If $N$ is the number of data points included in the analysis for a given liquid at one temperature and $n$ is the number of isotherms for this particular liquid, each minimum-slope observation was weighted by a factor $1 /(\mathrm{Nn})$ for this isotherm.

We find a slightly different result as we had previously observed for liquid dynamics controlled by temperature changes [18]. The histogram in Figure 2 a shows that the highfrequency relaxation of viscous liquids squeezed at constant temperature is most often characterized with $-0.45<\alpha_{\min }<-0.35$. In the previous study of the dielectric response of 52 liquids at ambient pressure, the most represented value interval for the minimum slope is around -0.5 , or more precisely, approximately $45 \%$ of the observed values of the minimum slope are $-0.55<\alpha_{\min }<-0.45$ [18]. The number of different chemicals is too small to conclude anything about the generality of this observation. Therefore, we have to look in more detail at how $\alpha_{\min }$ changes with compression, in order to find the trends.

Figure $3 \mathrm{a}$ shows the minimum slope evolution with loss peak frequency. It can be seen that it is similar to the temperature data at ambient pressure [17,18,31]; the high-frequency slope of the dielectric dissipation may converge slowly to $-\frac{1}{2}$ as pressure increases.
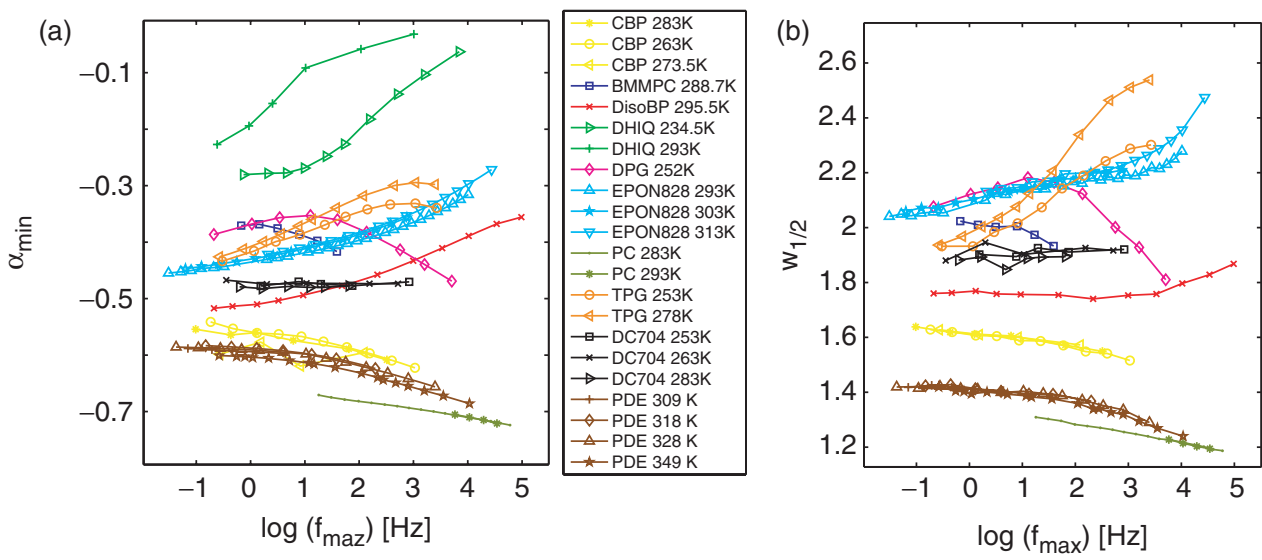

Figure 3. (Color online). (a): The minimum slope $\alpha_{\min }$ plotted as a function of pressure quantified by the position of the loss-peak frequency $f_{\max }$ for all nine liquids along isotherms. Every liquid has its own color. (b): The evolution of the half-width at half-maximum in decades write for the loss peak and normalized with the same quantity for a Debye process defined as $w_{\frac{1}{2}}=W_{\frac{1}{2}} / W_{D} / 2$, where $W_{\frac{1}{2}}$ is the number of decades of frequency on the right of the loss peak normalized with respect to the half Debye width $W_{D} / 2 \approx 0.572$ ( $w_{\frac{1}{2}} \rightarrow 1$ indicates Debye-like relaxation). In this plot the data points for PHIQ are absent because the corresponding loss at the frequency at half-maximum loss coincides with the $\beta$ relaxation loss. 
In an attempt to describe the shape of the $\alpha$ relaxation fully, we need a second shape parameter. We use the width, $W$, at half the dissipation maximum in decades. In an attempt to use as many datasets as possible and to avoid the effect of the DC-contribution to the left of the loss peak we use only the number of decades of the frequency on the right of the loss peak, $W_{\frac{1}{2}}$. From observations of different master curves, the following can be stated more or less generally: if there are changes in the shape of the loss, these are mainly due to changes of the slope of the high-frequency part. Thus, we can expect the same information about the shape from the full width and also from the width between the frequency at the loss peak and the highest frequency corresponding to half the loss. Here, we normalized the width $w_{\frac{1}{2}}$ with respect to the half Debye width $W_{D} / 2 \approx 0.572$. If $w_{\frac{1}{2}}=W_{\frac{1}{2}} / W_{D} / 2 \rightarrow 1$, then the relaxation is Debye-like. A plot of $w_{\frac{1}{2}}$ as a function of the frequency is shown in Figure 3b. The width follows qualitatively the behavior of $\alpha_{\min }$ to some extent, but it seems that it is less sensitive to temperature or pressure changes than the minimum slope.

Figure 3 is in agreement with findings regarding temperature-pressure superposition at same relaxation times, that the dielectric relaxation for some liquids depends only on the relaxation times and not on the temperature or pressure [6]. $\alpha_{\min }$, together with $w_{\frac{1}{2}}$, as functions of the frequency, describes the changes in the shape of the high-frequency dielectric loss at some relaxation time. If the minimum slope and width for two or more isotherms lie on top of each other, this means that the relaxation has the same shape for $f>f_{\max }$ to the inflection point. Thus, from this plot we can also estimate the frequency, below which the $\alpha$ relaxation (high-frequency part) is no longer affected by secondary processes that appear at times, and that are smaller than the $\alpha$-relaxation time and do not couple to the pressure and temperature in the same way as the primary process. This happens at a frequency where the curves of the shape quantities join into one; i.e. the relaxation time determines the shape of the relaxation and that is co-invariant of temperature or pressure changes [6].

The pressure-temperature superposition at the same relaxation time is relatively well obeyed in the case of the Van der Waals liquids PC (dark green), PDE (brown), and DC704 (black) if we look at both shape parameters $\alpha_{\min }$ and $w_{\frac{1}{2}}$. In contrast to the plot of TPG data (orange), it can be seen that the primary process shape is dominated by the secondary relaxation at pressures less than this; i.e. corresponding to loss peaks above $10 \mathrm{~Hz}$. In the case of PHIQ (light green), one can even see in Figure 3a that a TTPS is not observed in the measured frequency window. The same phenomenon is observed for PHIQ at ambient pressure [30] as well as under pressure [29] where even two types of secondary relaxations are identified. The dielectric relaxation for this liquid is characterized by a $\beta$ process with relatively high amplitude. For the other liquids with secondary processes above $1 \mathrm{kHz}$ we can see that deviations into $\alpha_{\min }$ isotherms are relatively small, and the $\beta$ process is characterized by low amplitude compared to the loss peak. It is clear that the temperature governs the $\beta$ process amplitude as well as the influence on the $\alpha_{\min }$ value: the higher the temperature, the larger $\alpha_{\min }$ at the same relaxation time. From this we can state that TTPS (and TTS) can only be observed if the influence of the $\beta$ process is relatively small. The spectrum for DC704 shows a secondary process with relatively low amplitude around $1 \mathrm{MHz}$. Thus, the $\alpha$ relaxation observed near $p_{\mathrm{g}}$ is well separated from $\beta$, and, therefore, $\alpha_{\min }=0.048$ is nearly constant over four frequency decades.

What about the liquids like PC and PDE? They have minimum slopes numerically bigger than $\frac{1}{2}$ and they are reported to include a hidden JG $\beta$ relaxation that is coupled to 
the $\alpha$ process and, therefore, the excess wings in the relaxation are invariant to pressure and temperature, when compared at a fixed value of the $\alpha$-relaxation time $[8,16]$.

Regarding the values of $\alpha_{\mathrm{min}}$, one might intuitively expect that interference from $\beta$ processes can only explain minimum slopes that are numerically smaller than $\frac{1}{2}$. Although for many years it was believed that secondary processes were found only in the $\mathrm{kHz}-\mathrm{MHz}$ frequency range, it is now generally recognized that these processes in some cases take place at much lower frequencies [28,32]. From measurements on liquids with a well-defined $\beta$ process in the $\mathrm{kHz}$ range, however, we and others (see, e.g. [33]) consistently find that when the liquid is cooled down to a temperature above the temperature where the $\alpha$ and $\beta$ processes merge, the high-frequency decay of the 'collapsed' $\alpha-\beta$ process has a minimum slope that is usually numerically larger than $\frac{1}{2}$. The same should be expected for the pressure-sensitive time-scale of the $\beta$ process. Thus, since whenever there are low-lying $\beta$ processes, the liquid is unavoidably around or above the $\alpha$ - $\beta$ merging temperature, or under the merging pressure, $\left|\alpha_{\min }\right|>1 / 2$ might occur as in the case of PC [8] or PDE [23]. This means that, in these liquids, a separation of the processes might not happen and thus TTPS is obeyed. PDE relaxation under $T_{\mathrm{g}}$ at ambient pressure shows two secondary processes [34]. If the dissipation is characterized with a well-defined $\beta$ process then $\alpha_{\min } \rightarrow-\frac{1}{2}$. In other words, 'genuine' $\alpha_{\min }=-\frac{1}{2}$ behavior only appears when the system is significantly below the merging temperature or above the merging pressure, as in the case of DC704.

An obvious question is whether the observed prevalence of minimum slopes around -0.4 is general or whether we should expect a value of -0.5 . If $\alpha_{\min }=-\frac{1}{2}$ was significant, one would expect that the closer the minimum slope is to $-\frac{1}{2}$, the better an inverse powerlaw description applies. This is investigated in Figure $4 \mathrm{a}$, which plots the third-order derivative relative to the first-order derivative, $\left|H^{(3)}\left(x_{0}\right) / \alpha_{\min }\right|$, where $H(x)=\log \varepsilon^{\prime \prime}(x)$, $x=\log f$, and $x_{0}$ is the $\log$ frequency at the point of the minimum slope. The idea is that, since the second-order derivative is zero at the frequency of the minimum slope, by
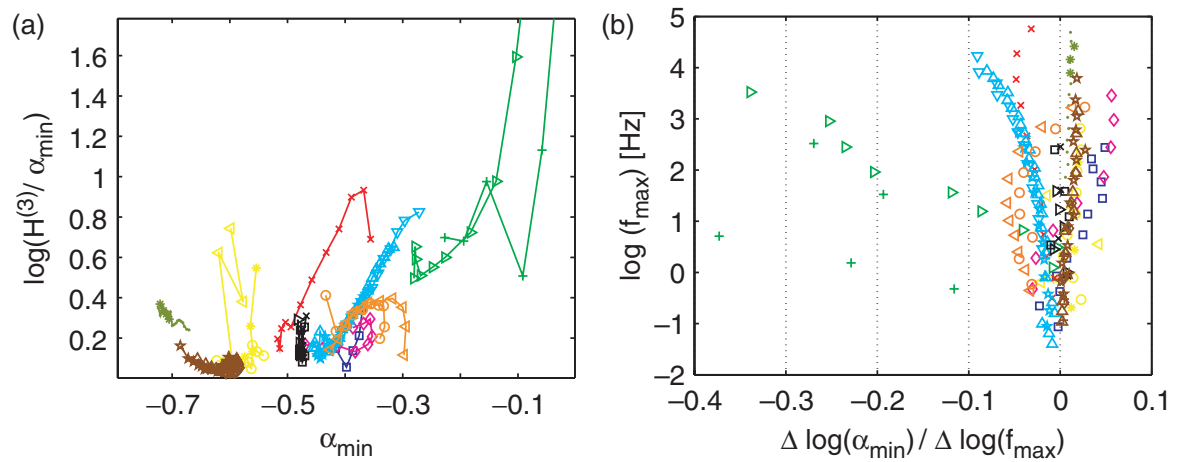

Figure 4. (Color online). (a): Third-order relative to first-order derivative, $\left.\mid H^{(3)}(x) / \alpha_{\min }\right) \mid$, at the frequency of the minimum slope for all datasets, where $H(x) \equiv \log \varepsilon^{\prime \prime}(x)(x=\log f)$. At the frequency of minimum slope the second-order derivative is zero; thus by Taylor's formula the smaller the thirdorder derivative is relative to the first-order derivative $\alpha_{\min }=H^{\prime}(x)$, the better an inverse power law description of the high-frequency loss applies. (b): The slope of $\alpha_{\min }$ gives information about how much $\alpha_{\min }$ is pressure-dependent. Both quantities contain information about the time-scales of the secondary processes. 
Taylor's formula the smaller $\left|H^{(3)}\left(x_{0}\right) / \alpha_{\min }\right|$, the frequency range is larger where the slope is almost constant. Figure $4 \mathrm{a}$ shows that the better an inverse power-law describes the loss, the closer $\alpha_{\min }$ is to $-\frac{1}{2}$ for DisBP (red + ) and EPON828 (cyan). For DC704 $\left|H^{(3)}\left(x_{0}\right) / \alpha_{\min }\right|$ lowers with pressure, while $\alpha_{\min }$ values are nearly constant, which means that $f^{\left|\alpha_{\min }\right|}$ applies to a bigger frequency range. The increase, and afterwards, decrease in $\left|H^{(3)}\left(x_{0}\right) / \alpha_{\min }\right|$ values (within the noise) captures the $\alpha-\beta$ merging process in the relaxation of BMMPC (blue) around $1 \mathrm{~Hz}$ and DPG (magenta) at $100 \mathrm{~Hz}$ (but DPG has more complex relaxation due to two $\beta$ processes $[35,36])$.

Let us again look at the information that we can extract from Figure $4 \mathrm{a}$ about the excess wing relaxations. For PDE the value of $\left|H^{(3)}\left(x_{0}\right) / \alpha_{\text {min }}\right|$ decreases with pressure increase, but it begins to increase slowly again. So, from a certain relaxation time (pressure) the frequency range of the $-\alpha_{\min }$ slope approximation begins to be smaller. This is an indication of a $\alpha-\beta$ merging process between 1 and $0.1 \mathrm{~Hz}$. This might be the case for CBP (yellow) as well. We cannot say anything consistent about the PC relaxation pattern. The experiental window is rather narrow. Confirmation of this observation for these liquids should be done on dielectric measurements at higher pressures.

The linearity of the minimum-slope pressure dependence can be seen in Figure $4 b$, which plots the change in slope point-by-point of the $\alpha_{\min }$ curves with the slowing of the relaxation. For DC704, which has a numerically minimum slope of $0.48, \alpha_{\min }$ is constant, while for all other liquids $\alpha_{\min }$ is changing in such a way that if $\alpha_{\min }$ approaches -0.5 , then its value is rather constant. It is interesting that materials with values of $\alpha_{\text {min }}$ numerically bigger than $\frac{1}{2}$, like for PC, have a minimum slope that changes very 'slowly' and almost linearly with $\log f$ over more than six decades. Thus, one can expect that, generally, $\alpha_{\min }$ will change until it reaches 0.5 and will be constant. One apparent exception is PDE; it seems to have a constant $\alpha_{\min }$ around -0.6 (Figures $4 \mathrm{~b}$ and $3 \mathrm{a}$ ), but together with Figure $4 \mathrm{a}$, this may be a sign of a hidden $\beta$ process at frequencies under $1 \mathrm{~Hz}$ that can contribute to the primary process as in the case of DPG.

In conclusion, the minimum slope and the width of the loss peak - shape parameters that describe only the high-frequency part of the dispersion - capture excellently the superposition of loss with the same relaxation times but at different temperatures and pressures. The considerable advantage of these two parameters is that they are model-free and thus independent of fitting procedures. With compression of the viscous liquid, the minimum slope value may converge to $-\frac{1}{2}$ (Figure 2a) as the $\alpha$ and $\beta$ processes separate (Figure 4a). The deviations from this power law are most likely due to interference from one or more secondary relaxation processes, with or without distinct maxima. If one or more secondary processes appear in the $\mathrm{Hz}$ range, it is practically impossible to separate the $\alpha$ and $\beta$ processes and this will be mirrored in the value of the minimum slope.

\section{Acknowledgements}

This work was supported by a grant from the Danish National Research Foundation (DNRF) for funding the Centre for Viscous Liquid Dynamics 'Glass and Time'.

\section{Notes}

1. If one believes in a simple additive relation between the primary relaxation and DC conductivity, then it is easy to show mathematically that $\varepsilon^{\prime \prime} \rightarrow \propto \omega^{-\beta}$ ) for large $\omega$. The cases 
where $\alpha$ process and DC decouple, but if all temperature curves are superimposed TTS is obeyed around the loss peak like in the situation for DisoBP. This means that the $\alpha$ process has no significant contribution from DC.

2. In our case, the construction of the capacitor for pressure investigations ensures an excellent separation of the sample from the pressure transmitting liquid - silicon oil. The observed DC conductivity results from some ions existing in every measured liquid irrespective of the purification procedure. The noise below $1 \mathrm{~Hz}$ is due to relatively small (below 0.1 ) values of $\varepsilon^{\prime \prime}$ of the presented samples and limitation experimental time.

\section{References}

[1] I. Gutzow and J. Schmelzer, The Vitreous State: Thermodynamics, Structure, Rheology, and Crystallization, Springer, Berlin, 1995.

[2] M.D. Ediger, C.A. Angell and S.R. Nagel, J. Phys. Chem. 100 (1996) p.13200.

[3] C.A. Angell, K.L. Ngai, G.B. McKenna et al., J. Appl. Phys. 88 (2000) p.3113.

[4] E. Donth, The Glass Transition, Springer, Berlin, 2001.

[5] J.C. Dyre, Rev. Mod. Phys. 78 (2006) p.953.

[6] K.L. Ngai, R. Casalini, S. Capaccioli et al., J. Phys. Chem. B 109 (2005) p.17356.

[7] K. Niss, C. Dalle-Ferrier, G. Tarjus et al., On the correlation between fragility and stretching in glassforming liquids, Available at arXiv:cond-mat/0611253 v1 (2006).

[8] S. Hensel-Bielowka, S. Pawlus, C.M. Roland et al., Phys. Rev. E 69 (2004) p.050501(R).

[9] M. Paluch, C.M. Roland, J. Gapinski et al., J. Chem. Phys. 118 (2003).

[10] D. Prevosto, S. Capaccioli, M. Lucchesi et al., J. Chem. Phys. 122 (2005) p.061102.

[11] K. Kessairi, S. Capaccioli, D. Prevosto et al., J. Phys. Chem. B 112 (2008) p.643.

[12] G.P. Johari and M. Goldstein, J. Chem. Phys. 53 (1970) p.2372.

[13] G.P. Johari, Ann. N.Y. Acad. Sci. 279 (1976) p.117.

[14] K.L. Ngai, J. Phys. Condens. Matter 15 (2003) p.S1107.

[15] G.P. Johari, G. Power and J.K. Vij, J. Chem. Phys. 116 (2002) p.5908.

[16] K.L. Ngai and M. Paluch, J. Chem. Phys. 120 (2004) p.857.

[17] N.B. Olsen, T. Christensen and J.C. Dyre, Phys. Rev. Lett. 86 (2001) p.1271.

[18] A. I. Nielsen et al. Submitted.

[19] S. Hensel-Bielowka, J. Zioło, M. Paluch et al., J. Chem. Phys. 117 (2002) p.2317.

[20] M. Paluch, J. Zioło, S.J. Rzoska et al., Phys. Rev. E 54 (1996) p.4008.

[21] K. Grzybowska, S. Pawlus, M. Mierzwa et al., J. Chem. Phys. 125 (2006) p.144507.

[22] M. Mierzwa, S. Pawlus, M. Paluch et al., J. Chem. Phys. 128 (2008) p.044512.

[23] S. Hensel-Bielowka and M. Paluch, Phys. Rev. Lett. 89 (2002) p.025704.

[24] M. Paluch, M. Sekula, S. Maslanka et al., J. Chem. Phys. 120 (2004) p.2020.

[25] C.M. Roland, S. Hensel-Bielowka, M. Paluch et al., Rep. Prog. Phys. 68 (2005) p.1405.

[26] F. Kremer and A. Schönhals, eds., Broadband Dielectric Spectroscopy, Springer, Berlin, 2002.

[27] A. Kudlik, S. Benkhof, T. Blochowicz et al., J. Mol. Struct. 479 (1999) p.201.

[28] N.B. Olsen, J. Non-Cryst. Solids 235 (1998) p.399.

[29] M. Paluch, S. Pawlus, S. Hensel-Bielowka et al., J. Phys. Rev. B 72 (2005) p.224205.

[30] R. Richert, K. Duvvuri and L.-T. Duong, J. Chem. Phys. 118 (2003) p.1828.

[31] B. Jakobsen, K. Niss and N.B. Olsen, J. Chem. Phys. 123 (2005) p.234511.

[32] U. Schneider, R. Brand, P. Lunkenheimer et al., Phys. Rev. Lett. 84 (2000) p.5560.

[33] L.-M. Wang and R. Richert, Phys. Rev. B 76 (2007) p.064201.

[34] S. Kahle, J. Gapinski, G. Hinze et al., J. Chem. Phys. 122 (2005) p.074506.

[35] R. Casalini and C.M. Roland, Phys. Rev. Lett. 91 (2003) p.015702.

[36] R. Casalini and C.M. Roland, Phys. Rev. B 69 (2004) p.094202. 